\title{
Ion Channeling Contrast Imaging of Aluminum Wire Bonds
}

\author{
Karen D. Dye and Robert A. Youngman \\ Medtronic, Inc., Microelectronics Center, Tempe, AZ 85284
}

Ion channeling contrast, as developed during scanning ion microscopy, has been established as a reliable technique for the determination of grain size [1]. This paper extends the technique to the evaluation of wirebond microstructure. The effect of bonding parameters on grain size and morphology is evaluated and evidence of localized heating is observed.

Bond formation in metallic solids can occur by diffusion when metal atoms acquire enough energy to overcome the activation barrier. In ultrasonic wirebonding, the bond is formed by introduction of ultrasonic energy while applying a clamping force. It has been proposed that the ultrasonic "scrubbing" action breaks up surface oxides and contaminants, allowing atomically clean metals to come in direct contact and form a bond at relatively low temperatures (well below the melting temperatures of the constituents). It is also proposed that localized heating during the process is not sufficient to play a substantial role in the bonding process [2]. Ultrasonic power, clamping pressure, and time are the key process variables. In this study, bonds formed undertwo sets of parameters were evaluated. Test sample preparation consisted of mounting Si die on PWB hybrids and bonding with 32-micrometer diameter aluminum (Al 1\% Si) wire under two conditions.

Samples of bonded die were encapsulated in epoxy and metallurgically crosssectioned. Conventional analysis of such Al microstructures utilizing chemical etchants was unsuccessful in revealing any reliable information on the Al grain size and other microstructural features. Unetched versions of the same samples were placed in a FEI 800xP FIB and ion channeling contrast images of the wirebonds were collected [3]. The ion channeling contrast micrograph of Fig. 1 clearly shows delineation of the $\mathrm{Al}$ grains present in the wire and the Si die metallization. The grain size of the bonding wire is much finer than that of the die metallization. Grain size analysis utilizing image analysis yields an average grain diameter of 0.7 micrometers for the wire and 2.9 micrometers for the metallization. Low magnification FIB images showing cross sections through wirebonds typical of the two process conditions are shown in Figs. 2-3. Note that although the bond widths are approximately equal, the ion channeling micrographs reveal striking differences in the relative amounts of bond penetration. Condition A shows appreciably deeper penetration of the bond and more material accumulated at bond edges. Upon examination at higher magnifications, it is apparent that the accumulated material at bond edges is due to substantial deformation of the grains (Fig. 4). This deformation is most pronounced in bonds made using condition A. Perhaps the most striking feature observed is evidence of recrystallization of the pad metal. The grain size of the metallization in the region of the bond is significantly smaller than that observed elsewhere on the die. This is indicative of substantial localized heating during wire bonding. Further studies including variations in all wirebond parameters are underway.

FIB ion channeling contrast has been utilized for evaluation of the details of the microstructure of Al wirebonds. Contrary to accepted understanding of the nature of the wirebond process; localized heating has been identified as a possible factor in the bonding mechanism. 


\section{References}

[1] K. Nikawa, et al., 1989 International Reliability Physics Symposium, 27 (1989), 43-51.

[2] G. Harman, Wire Bonding in Microelectronics, McGraw-Hill, New York, 1997.

[3] FEI Technical Note, XP Grain Size Analysis, PN 21851.
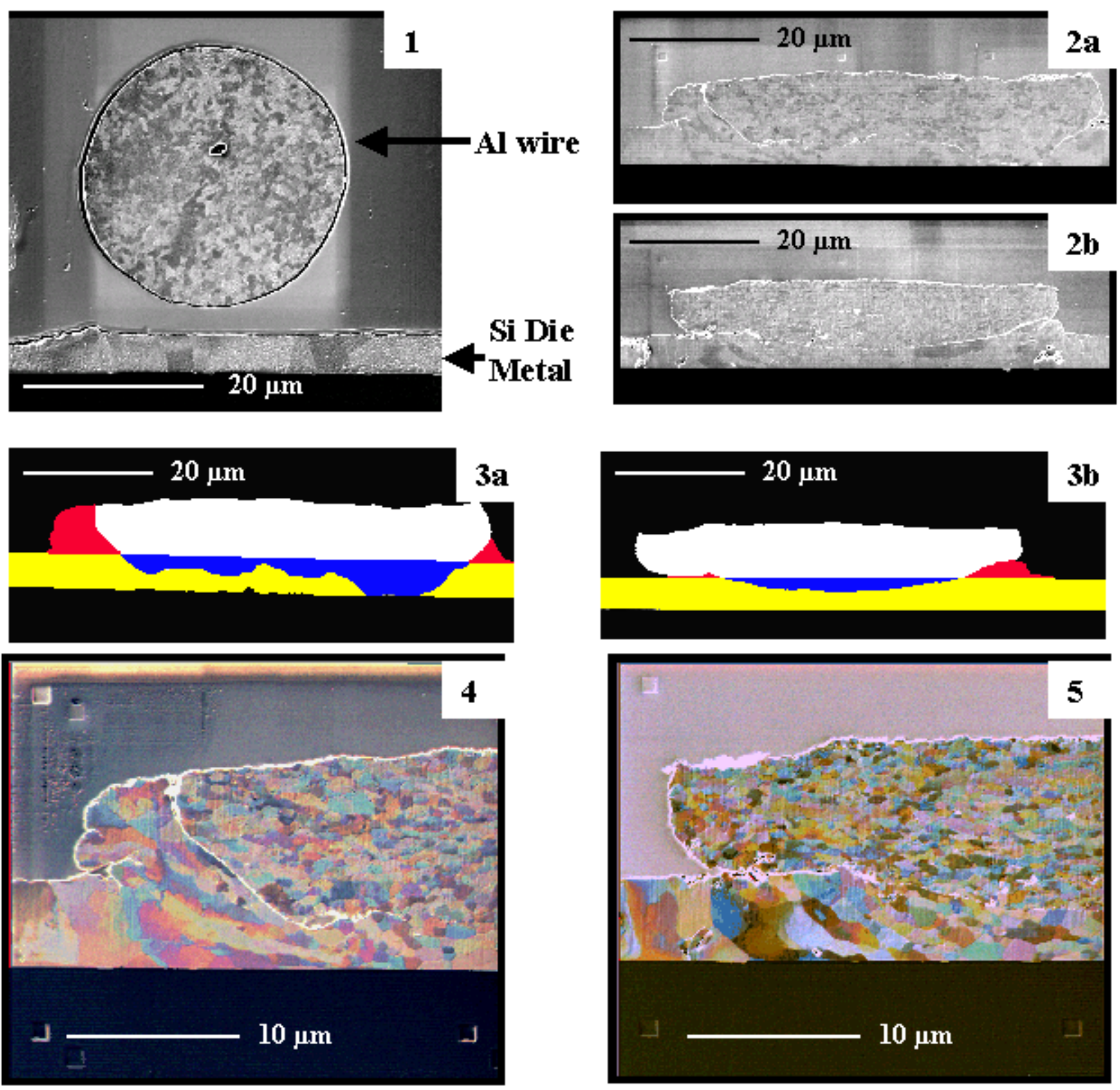

FIG. 1. SE FIB image: cross-section through wire and bonding pad.

FIG. 2a. SE FIB image: cross-section through typical bond - Condition A (60 KHz, 30g, 20msec).

FIG. 2b. SE FIB image: cross-section through typical bond-Condition B (120 KHz, 30g, 10msec).

FIG. 3a. Colorized SE FIB image: bond shown in Fig. 2a (Condition A).

FIG. 3b. Colorized SE FIB image: bond shown in Fig. 2b (Condition B).

FIG. 4. RGB overlay of 3 tilts: higher magnification view of bond shown in Fig. 2a (Condition A).

FIG. 5. RGB overlay of 3 tilts: higher magnification view of bond shown in Fig. 2b (Condition B). 